\title{
JIG \& FIXTURE DESIGN FOR ROUGHNESS TESTER SJ-210
}

\author{
Nanda Rahmaniar Siswanda, Sufiyanto*, Sudjatmiko
}

Department of Mechanical Engineering, Faculty of Engineering, University of Merdeka Malang

*Email corresponding author: sufiyanto@ unmer.ac.id

\begin{abstract}
The rapid development of the industry gave rise to better technological innovations to develop the quality capacity of a production. Jig \& fixture is a production tool used in the manufacturing process, so that accurate part duplication is produced. Jigs \& fixture specifically designed to facilitate the setup of materia; which ensures uniformity in the shape and size of products in large quantities (mass products), and commemorate the time of production [2-4]. Jig \& fixture functions to hold and direct the workpiece, so that the manufacturing process is more efficient and product quality can be maintained as specified quality. Rong and Zhu (1999) in Ahmad Rizki (2003) state that an object consists of several surface surfaces. In the use of a fixture, the placement process (locating) is the process of placing multiple surfaces of a workpiece until it touches the locator, which is then followed by the clamping process of the workpiece that comes in contact with the locator called a locating surface. In a workpiece there are 6 degrees of freedom (degree of freedom) of movement, ie linear motion in the direction or counterclockwise with the $X, Y, Z$ axis and rotational movements towards the $X, Y$, and $Z$ axis in a clockwise or counterclockwise direction.
\end{abstract}

Keywords : locator, clamping, degree of freedom

\section{INTRODUCTION}

The rapid development of the industry has given rise to better technological innovations to develop the capacities of the quality of production. In order to reduce the production wing, increasing the efficiency of the manufacturing process of a product is very influential, especially by reducing the time of the manufacturing process. Almost the entire production process uses machines that match the specifications of the product being made. The more complex the product, the more complicated the tools used. Improving product quality can only be achieved by using production process aids, such as jigs \& fixtures, molds, and dies.

Jigs \& fixtures are production tools used in the manufacturing process, so that accurate part duplication results. The jig \& fixture is specially designed to simplify the setting of material; which guarantees the diversity of shapes and sizes of the product in large quantities (mass product), as well as reducing the timing of production. Jig \& fixture functions to hold and direct the work work, so that the manufacturing process is more efficient and the quality of the product can be maintained according to a predetermined quality.

According to Edgrand G. Hoffman (1996), jigs \& fixtures are production tools used in the manufacturing process, resulting in accurate part duplication. Jigs \& fixtures are usually made specifically as a means of assisting the production process to simplify the setting of materials which ensure the diversity of shapes and sizes of the product in large quantities (mass product) and to shorten the production time. Jigs \& fixtures are one type of auxiliary tool found in the manufacturing process so that they are obtained by a uniform product with high accuracy.

Rongdan Zhu (1999) in Ahmad Rizki (2003) states that a bendater consists of several 
surfaces. In the use of a fixture, the process of placing (locating) is the process of placing multiple surfaces of the workpiece until it is in contact with the locator, which is then followed up by the clamping process of the workpiece in contact with the locator is called the locating surface. In a work, there are 6 degrees of freedom of movement, which is linear movement in the direction of or against direction of the $\mathrm{X}, \mathrm{Y}, \mathrm{Z}$ axes and the movement of the rotation to the $\mathrm{X}, \mathrm{Y}$, and $\mathrm{Z}$ axes or against the clockwise direction.

\section{Research Question}

Based on the background of the problems how to design a Jig \& Fixture for the SJ-210 Roughness Tester?

\section{Research Limitation}

As for the research, it will limit the problems to its parts, namely:

1. The discussion focused on the design of the Jig \& Fixture for the SJ-210 Roughness Tester

2. Process on work using conventional welding and milling machines

3. The process of making the clamping board

\section{METHODOLOGY OF RESEARCH}

\section{Research Flowchart}

The following is research flowchart for this study.

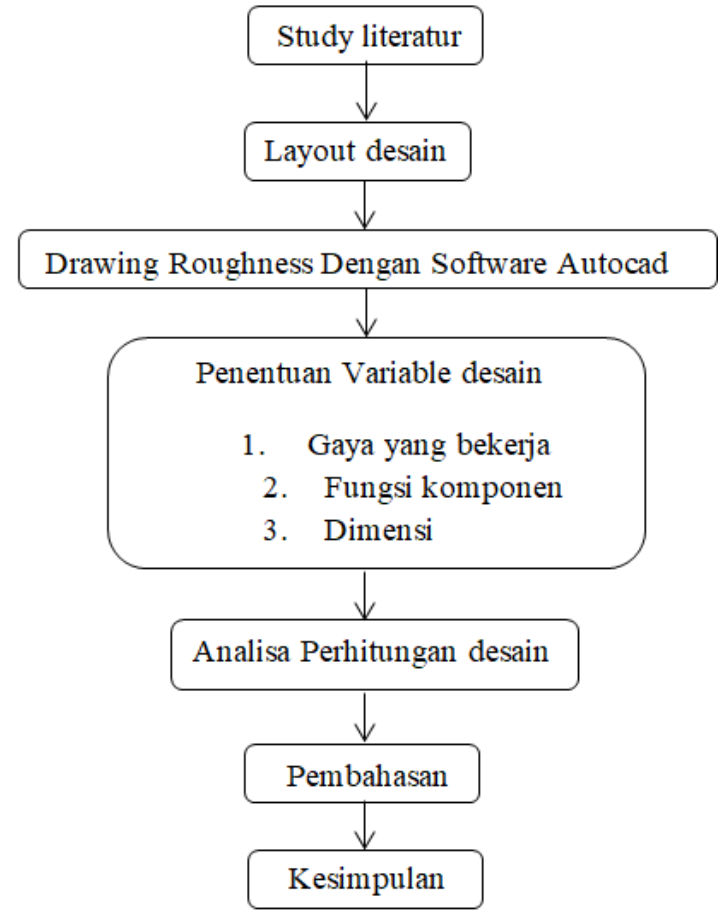

Figure 1. Research Flowchart

\section{Tools and Materials}

The tools used in this research are as follows:

1. Thread

2. Bolt

3. Bearings

4. Vise

5. Cast Steel Rod

While the materials used in this study are as follows:

1. S45C Steel (For Shaft Holder and plate clamp)

2. Bearing no.6001 $(\mathrm{d} 12, \mathrm{D}=28, \mathrm{~b}=8, \mathrm{r}=$ $0.5, \mathrm{c}=400, \mathrm{co}=229$ )

3. Steel bolt 


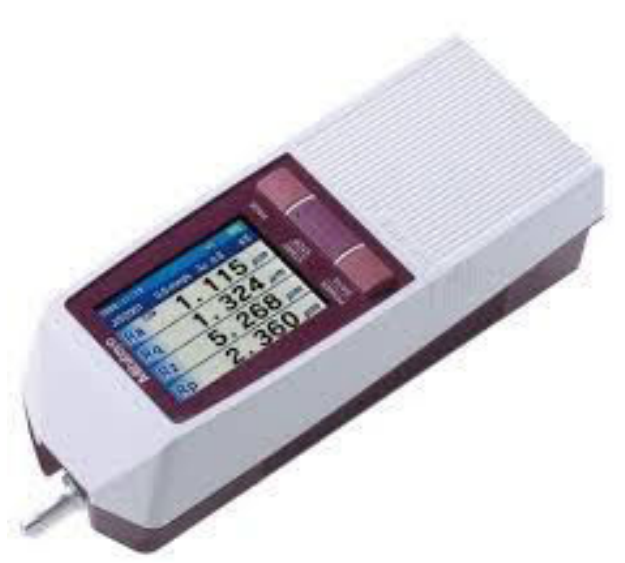

Figure 2. Roughness Tester SJ-210

\section{Tools Specification}

a. The 2.4-inch color graphic LCD provides excellent readability and an intuitive display that is easy to negotiate. The LCD also includes a flat cover for improved visibility in dark environments.

b. The Surftest SJ-210 can be operated easily using the buttons on the front of the unit and below the slide cover.

c. Up to 10 measurement conditions and measured profiles can be stored in the internal memory.

d. Memory cards can be used as additional memory to store a large number of the profile and conditions being measured.

e. Access to each feature can be password protected, which prevents undesired operations and allows you to protect your settings.

f. The interface supports 16 languages, which can be changed freely.

g. An alarm warns you when the cumulative measurement distance exceeds the set limit. h. Surftest SJ-210 meets the following standards: JIS (JIS-B0601-2001, JISB0601-1994, JIS B0601-1982), VDA, ISO1997, and ANSI.

\section{Design}

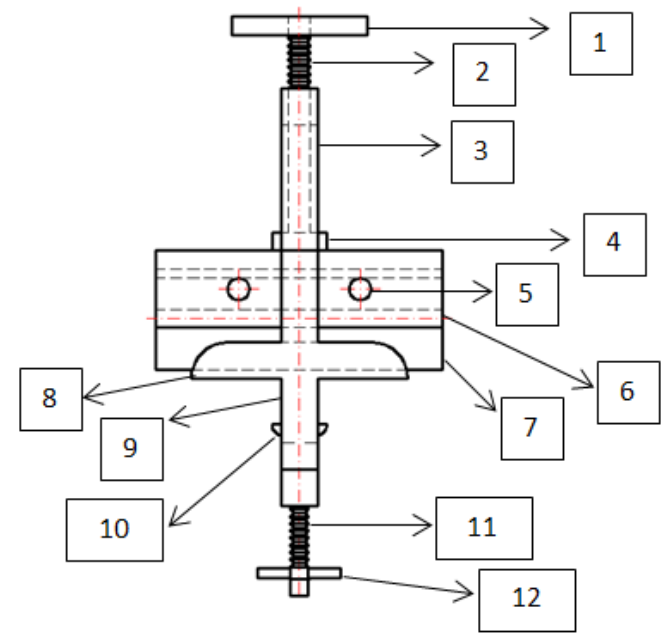

Keterangan :
1. Setir
7. Roughness tester
2. Ulir setir
8. Dudukan
3. Lengan dudukan
9. Lengan ragum
4. Bantalan
10. Penahan Ragum
5. Mur baut
11. Ulir ragum
6. Catokan Roughness tester
12. Tuas Ragum

\section{Side View Image}

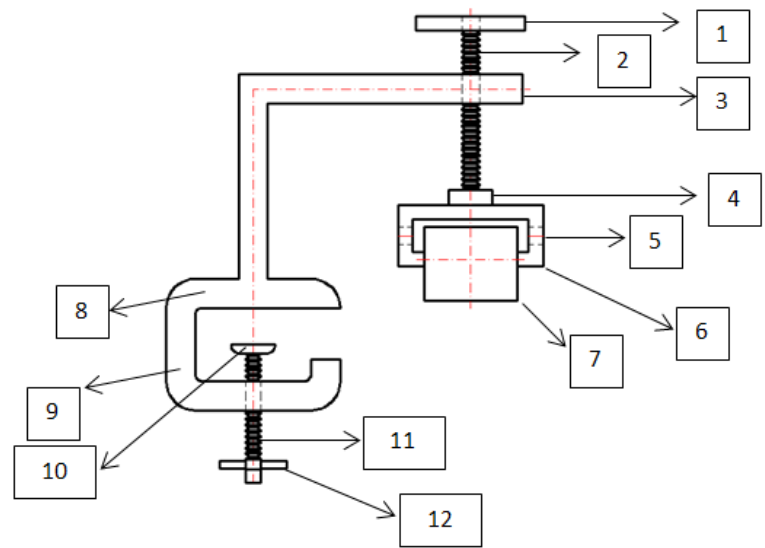


Keterangan :
1. Setir
2. Ulir setir
3. Lengan dudukan
4. Bantalan
5. Murbaut
6. Catokan Roughness tester

7. Roughness test

8. Dudukan

9. Lengan ragum

10. Penahan Ragul

11. Ulir ragum

12. Tuas Ragum

Bearing no. $6001(\mathrm{~d}=12, \mathrm{D}=28, \mathrm{~b}=8, \mathrm{r}=$ $0.5, \mathrm{c}=400$, co $=229$ ).

Jig arms and fixtures $2 \mathrm{~cm}$ (plow plate S45C).

Drive 400 grams.

Power: $25 \mathrm{~kg}$ area

Vise holder 200 grams

Lever Vise 200 grams

\section{Front View Image}

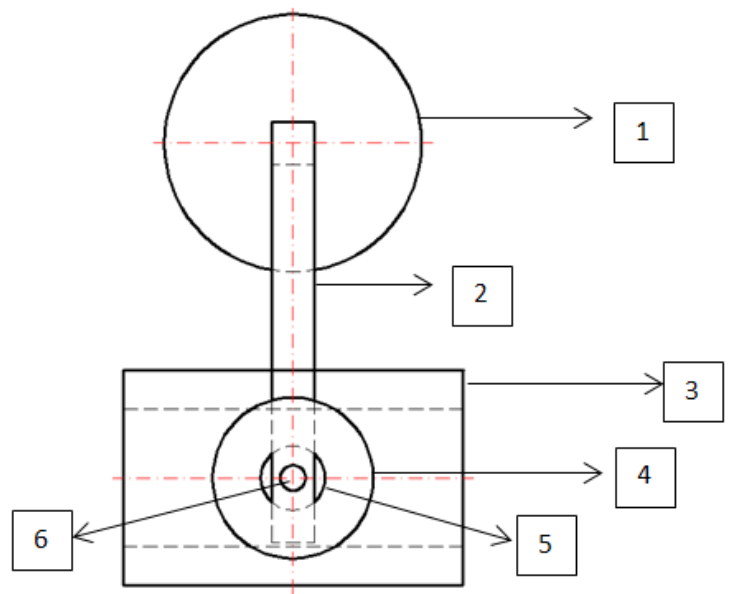

Keterangan :

1. Dudukan

2. Lengan Dudukan

3. Pencatok Roughness Tester

4. Setir

5. Bantalan

6. Ulir setir

\section{Calculation}

\section{Planned equipment specifications:}

Thread diameter $10 \mathrm{~mm}$ (made of S45C steel) coarse symmetrical thread at tabled $1=8.376 \mathrm{~mm}, \mathrm{~d} 2=9.026 \mathrm{~mm}, \mathrm{D}=10$ $\mathrm{mm}, \mathrm{p}=1.5 \mathrm{~mm}, \mathrm{H} 1=0.812 \mathrm{~mm}$, thread diameter $12 \mathrm{~mm}$ (made of $\mathrm{S} 45 \mathrm{C}$ steel) rough thread $\mathrm{d} 2=10.863 \mathrm{~mm}, \mathrm{D}=12 \mathrm{~mm}, \mathrm{p}=1.75$ $\mathrm{mm}, \mathrm{H} 1=0.947 \mathrm{~mm}$.

$7 \mathrm{~mm} \mathrm{S45C} \mathrm{plate} \mathrm{roughness} \mathrm{tester.}$

\section{a. Thread Weight Diameter 12 with a length of $127 \mathrm{~mm}$}

$16 \mathrm{~mm} \times 6000 \mathrm{~mm}=9,5 \mathrm{~kg}$

$12 \mathrm{~mm} \times 127 \mathrm{~mm}=\mathrm{X}$

$96000 \mathrm{~mm}^{2}=9,5 \mathrm{~kg}$

$1524 \mathrm{~mm}^{2}=\mathrm{X}$

$X=>\frac{1524 \mathrm{~mm}^{2} \times 9,5}{96000 \mathrm{~mm}^{2}}=\frac{14478 \mathrm{~kg}}{96000}$

$=0,15 \mathrm{~kg}=150$ gram

\section{b. Thread Weight Diameter 12 with a length of $73 \mathrm{~mm}$}

$16 \mathrm{~mm} \times 6000 \mathrm{~mm}=9,5 \mathrm{~kg}$

$12 \mathrm{~mm} \times 73 \mathrm{~mm}=\mathrm{X}$

$96000 \mathrm{~mm}^{2}=9,5 \mathrm{~kg}$

$876 \mathrm{~mm}^{2}=\mathrm{X}$

$X=>\frac{876 \mathrm{~mm}^{2} \times 9,5}{96000 \mathrm{~mm}^{2}}=\frac{8322 \mathrm{~kg}}{96000}$

$=0,08 \mathrm{~kg}=80$ gram

\section{c. Thread Weight Diameter 10 with a length of $30 \mathrm{~mm}$}

$16 \mathrm{~mm} \times 6000 \mathrm{~mm}=9,5 \mathrm{~kg}$

$10 \mathrm{~mm} \times 30 \mathrm{~mm}=\mathrm{X}$

$96000 \mathrm{~mm}^{2}=9,5 \mathrm{~kg}$

$300 \mathrm{~mm}^{2}=\mathrm{X}$

$X=>\frac{1524 \mathrm{~mm}^{2} \times 9,5}{96000 \mathrm{~mm}^{2}}=\frac{14478 \mathrm{~kg}}{96000}$

$=0,15 \mathrm{~kg}=150$ gram 


\section{Iron weight, roughness tester}

A black steel plate $100 \times 160=1$ piece

$43 \times 160=2$ pieces

$8 \times 160=2$ pieces

$6 \mathrm{~mm} \times 1200 \mathrm{~mm} \times 2400 \mathrm{~mm}=140 \mathrm{~kg}$

$6 \mathrm{~mm} \times 100 \mathrm{~mm} \times 160 \mathrm{~mm}=\mathrm{X}$

$17280000 \mathrm{~mm}^{3}=140 \mathrm{~kg}$

$96000 \mathrm{~mm}^{3}=\mathrm{X}$

$X=>\frac{96000 \mathrm{~mm}^{3} \times 140 \mathrm{~kg}}{17280000 \mathrm{~mm}^{3}}=\frac{14478 \mathrm{~kg}}{1728}$

$=0,7 \mathrm{~kg}=700$ gram

$6 \mathrm{~mm} \times 1200 \mathrm{~mm} \times 2400 \mathrm{~mm}=140 \mathrm{~kg}$

$6 \mathrm{~mm} \times 43 \mathrm{~mm} \times 160 \mathrm{~mm}=\mathrm{X}$

$17280000 \mathrm{~mm}^{3}=140 \mathrm{~kg}$

$41280 \mathrm{~mm}^{3}=\mathrm{X}$

$X=>\frac{41280 \mathrm{~mm}^{3} \times 140 \mathrm{~kg}}{17280000 \mathrm{~mm}^{3}}=\frac{57792 \mathrm{~kg}}{172800}$

$=0,3 \mathrm{~kg}=300$ gram

$=300$ gram $\times 2=600$ gram

$6 \mathrm{~mm} \times 1200 \mathrm{~mm} \times 2400 \mathrm{~mm}=140 \mathrm{~kg}$

$6 \mathrm{~mm} \times 8 \mathrm{~mm} \times 160 \mathrm{~mm}=\mathrm{X}$

$17280000 \mathrm{~mm}^{3}=140 \mathrm{~kg}$

$7680 \mathrm{~mm}^{3}=\mathrm{X}$

$X=>\frac{7680 \mathrm{~mm}^{3} \times 140 \mathrm{~kg}}{17280000 \mathrm{~mm}^{3}}=\frac{10752 \mathrm{~kg}}{172800}$

$=0,06 \mathrm{~kg}=60 \mathrm{gram}$

$=60$ gram $\times 2=120$ gram

The total weight of the roughness tester $=700$

gram +600 gram +120 gram $=1,42 \mathrm{Kg}$

\section{Weight arm straightener}

Iron steel black plate $100 \times 160=1$ piece

$43 \times 160=2$ pieces

$20 \mathrm{~mm} \times 1200 \mathrm{~mm} \times 2400 \mathrm{~mm}=467 \mathrm{~kg}$

$20 \mathrm{~mm} \times 100 \mathrm{~mm} \times 160 \mathrm{~mm}=\mathrm{X}$

$57600000 \mathrm{~mm}^{3}=467 \mathrm{~kg}$

$72000 \mathrm{~mm}^{3}=\mathrm{X}$
$X=>\frac{72000 \mathrm{~mm}^{3} \times 467 \mathrm{~kg}}{57600000 \mathrm{~mm}^{3}}=\frac{33624 \mathrm{~kg}}{57600}$

$=0,6 \mathrm{~kg}=600$ gram

$20 \mathrm{~mm} \times 1200 \mathrm{~mm} \times 2400 \mathrm{~mm}=467 \mathrm{~kg}$

$20 \mathrm{~mm} \times 120 \mathrm{~mm} \times 20 \mathrm{~mm}=\mathrm{X}$

$57600000 \mathrm{~mm}^{3}=467 \mathrm{~kg}$

$72000 \mathrm{~mm}^{3}=\mathrm{X}$

$X=>\frac{48000 \mathrm{~mm}^{3} \times 467 \mathrm{~kg}}{57600000 \mathrm{~mm}^{3}}=\frac{22416 \mathrm{~kg}}{57600}$

$=0,4 \mathrm{~kg}=400$ gram

\section{Weight of Circle Standing}

$20 \mathrm{~mm}$ x $1200 \mathrm{~mm} \times 2400 \mathrm{~mm}=467 \mathrm{~kg}$

$20 \mathrm{~mm} \times 3,14 \mathrm{~mm} \times 60^{2} \mathrm{~mm}=\mathrm{X}$

$57600000 \mathrm{~mm}^{3}=467 \mathrm{~kg}$

$226080 \mathrm{~mm}^{3}=\mathrm{X}$

$\mathrm{X}=>\frac{226080 \mathrm{~mm}^{3} \times 467 \mathrm{~kg}}{57600000 \mathrm{~mm}^{3}}=\quad \frac{10557936 \mathrm{~kg}}{5760000}$

$=1,8 \mathrm{~kg}=1800$ gram

\section{Weight of Vise}

$20 \mathrm{~mm} \times 1200 \mathrm{~mm} \times 2400 \mathrm{~mm}=467 \mathrm{~kg}$

$20 \mathrm{~mm} \times 50 \mathrm{~mm} \times 20 \mathrm{~mm}=\mathrm{X}$

$57600000 \mathrm{~mm}^{3}=467 \mathrm{~kg}$

$20000 \mathrm{~mm}^{3}=\mathrm{X}$

$X=>\frac{20000 \mathrm{~mm}^{3} \times 467 \mathrm{~kg}}{57600000 \mathrm{~mm}^{3}}=\frac{934 \mathrm{~kg}}{57600}$

$=0,16 \mathrm{~kg}=160$ gram

$20 \mathrm{~mm} \times 1200 \mathrm{~mm} \times 2400 \mathrm{~mm}=467 \mathrm{~kg}$

$20 \mathrm{~mm} \times 120 \mathrm{~mm} \times 20 \mathrm{~mm}=\mathrm{X}$

$57600000 \mathrm{~mm}^{3}=467 \mathrm{~kg}$

$72000 \mathrm{~mm}^{3}=\mathrm{X}$

$X=>\frac{48000 \mathrm{~mm}^{3} \times 467 \mathrm{~kg}}{57600000 \mathrm{~mm}^{3}}=\frac{22416 \mathrm{~kg}}{57600}$

$=0,4 \mathrm{~kg}=400$ gram

$20 \mathrm{~mm} \times 1200 \mathrm{~mm} \times 2400 \mathrm{~mm}=467 \mathrm{~kg}$

$20 \mathrm{~mm} \times 15 \mathrm{~mm} \times 20 \mathrm{~mm}=\mathrm{X}$

$57600000 \mathrm{~mm}^{3}=467 \mathrm{~kg}$

$6000 \mathrm{~mm}^{3}=\mathrm{X}$ 
$X=>\frac{6000 \mathrm{~mm}^{3} \times 467 \mathrm{~kg}}{57600000 \mathrm{~mm}^{3}}=\frac{2802 \mathrm{~kg}}{57600}$

$=0,05 \mathrm{~kg}=50$ gram

Total weight o vise 160 gram +400 gram +50

gram $=610$ gram

\section{Bolt nut (Locking Roughness Tester)}

Thread diameter $10 \mathrm{~mm}$ (S45C steel) rough symmetrical thread on the table

$\mathrm{d}_{1}=8,376 \mathrm{~mm}, \mathrm{~d}_{2}=9,026 \mathrm{~mm}$,

$\mathrm{D}=10 \mathrm{~mm}, \mathrm{p}=1,5 \mathrm{~mm}, \mathrm{H}_{1}=0,812 \mathrm{~mm}$.

\section{RESULT AND DISCUSSION}

\section{a. Tensile of Thread}

$\sigma \mathrm{t}=\frac{\mathrm{w}}{\mathrm{A}}=\frac{\mathrm{w}}{\frac{\mathrm{m}}{4} \cdot \mathrm{d} 1^{2}}\left(\mathrm{~kg} / \mathrm{mm}^{2}\right)$

(Sularso's book p 296 no 7.1 )

Where: $\sigma_{\mathrm{t}}=$ tensile stress $\left(\mathrm{kg} / \mathrm{mm}^{2}\right)$.

$$
\mathrm{W}=\text { axial tensile load on the }
$$
bolt $(\mathrm{kg})$.

$$
\sigma \mathrm{t}=\frac{\mathrm{W}}{\frac{\pi}{4} \cdot \mathrm{d1}^{2}}
$$

$$
=\frac{1,82 \mathrm{~kg}}{\frac{3,14}{4} \cdot 8,376 \mathrm{~mm}^{2}}
$$

$=3,3 \mathrm{~kg} / \mathrm{mm}^{2}$

\section{b. Shear stress permission}

$\tau_{\mathrm{a}}=(0,5 \mathrm{~s} . d \mathrm{0}, 75) \sigma_{\mathrm{a}}\left(\mathrm{kg} / \mathrm{mm}^{2}\right)$

(Sularso's book p 297 )

Where: $\tau_{\mathrm{a}}=$ shear stress permission $\left(\mathrm{kg} / \mathrm{mm}^{2}\right)$

$\tau_{\mathrm{a}}=0,5 \times 4,8 \mathrm{~kg} / \mathrm{mm}^{2}$

$=2,4 \mathrm{~kg} / \mathrm{mm}^{2}$

\section{c. Number of Nut Threads}

$$
\mathrm{Z} \geqq \frac{W}{\pi d_{2} H_{1} q_{a}}
$$

(Sularso's book p 297 no.7.5)

where: $\mathrm{H}_{1}=$ height of hook $(\mathrm{mm})$

$$
\begin{aligned}
\mathrm{Z} & =\frac{1,82 \mathrm{~kg}}{3,14 \times 9,026 \mathrm{~mm} \times 0,812 \mathrm{~mm} \times 3 \frac{\mathrm{kg}}{\mathrm{mm} 2}} \\
& =3
\end{aligned}
$$

\section{d. Nut Height}

$$
\mathbf{H}=\mathbf{z} \cdot \mathbf{p}(\mathbf{m m})
$$

where: $\mathrm{H}=$ nut height $(\mathrm{mm})$

standard: $H=(0,8$ s.d 1,0$) . d(m m)$

$\mathrm{p}=$ distance divider $(\mathrm{mm})$

(look at the table of 7.1. a \& 7.1. b \& table7.2 Sularso's book p 289-291)

$$
\begin{aligned}
\mathbf{H} & =3 \times 2,5 \mathrm{~mm} \\
& =7,5 \mathrm{~mm}
\end{aligned}
$$

\section{e. Thread Root Shear Stress on Bolt}

$$
\tau_{b}=\frac{w}{\pi . d_{1} \cdot k \cdot p \cdot z} \quad\left(\mathrm{~kg} / \mathrm{mm}^{2}\right)
$$

(Sularso's book p 297 no 7.8 )

where: $\tau_{\mathrm{b}}=$ thread root shear stress on the bolt $\left(\mathrm{kg} / \mathrm{mm}^{2}\right)$

$\mathrm{k} . \mathrm{p}=$ outer root thread thickness

$$
\begin{aligned}
& \mathrm{k}=0,84 \\
& \tau_{b}=\frac{1.82 \mathrm{~kg}}{3,14 \times 8,376 \mathrm{~mm} \times 0,84 \times 1,5 \mathrm{~mm} \times 3}
\end{aligned}
$$

\section{f. Thread Root Shear Stress in Nuts}

$$
\tau_{\mathrm{n}}=\frac{\mathrm{w}}{\pi . \text {.j.p.z }} \quad\left(\mathrm{kg} / \mathrm{mm}^{2}\right)
$$

(Sularso's book p 297 no. 7.9)

where: $\tau_{n}=$ thread root shear stress on

$$
\begin{gathered}
\text { the nut }\left(\mathrm{kg} / \mathrm{mm}^{2}\right) \\
\text { j }=0,75 \text { (symmetrical thread) } \\
\text { Sularso's book p. } 297
\end{gathered}
$$

$$
\begin{aligned}
\tau_{\mathrm{n}} & =\frac{1,82 \mathrm{~kg}}{3,14 \times 10 \mathrm{~mm} \times 0,75 \times 1,5 \mathrm{~mm} \times 3} \\
\tau_{\mathrm{n}} & =2 \mathrm{~kg} / \mathrm{mm}^{2}
\end{aligned}
$$

Thread (Vise Roughness Tester) Thread diameter $12 \mathrm{~mm}$ (made of S45C steel) coarse metric thread on the table $\mathrm{d}_{1}=10,106 \mathrm{~mm}, \mathrm{~d}_{2}=10,863 \mathrm{~mm}$, $\mathrm{D}=12 \mathrm{~mm}, \mathrm{p}=1,75 \mathrm{~mm}, \mathrm{H}_{1}=0,947$ $\mathrm{mm}$.

\section{a. Thread Tensile Stress}

$$
\sigma \mathrm{t}=\frac{\mathrm{w}}{\mathrm{A}}=\frac{\mathrm{w}}{\frac{\pi}{4} \cdot \mathrm{d1}^{2}}\left(\mathrm{~kg} / \mathrm{mm}^{2}\right)
$$

(Sularso's book p 296 no 7.1 )

where $: \sigma_{\mathrm{t}}=$ tensile stress $\left(\mathrm{kg} / \mathrm{mm}^{2}\right)$. 


$$
\begin{aligned}
& \mathrm{W}=\text { axial tensile load on the } \\
& \text { bolt }(\mathrm{kg}) \text {. } \\
& \sigma \mathrm{t}=\frac{\mathrm{W}}{\frac{\pi}{4} \cdot \mathbf{d 1 ^ { 2 }}} \\
& =\frac{1,82 \mathrm{~kg}}{\frac{3,14}{4} \cdot 10,106 \mathrm{~mm}^{2}} \\
& =2,3 \mathrm{~kg} / \mathrm{mm}^{2}
\end{aligned}
$$

\section{b. Shear stress permission}

$$
\tau_{\mathrm{a}}=\left(\begin{array}{llll}
0,5 \mathrm{~s} . \mathrm{d} & 0,75) & \sigma_{\mathrm{a}} & \left(\mathrm{kg} / \mathrm{mm}^{2}\right)
\end{array}\right.
$$

(Sularso's book p 297 )

where: $\tau_{\mathrm{a}}=$ shear stress permission

$$
\begin{aligned}
\left(\mathrm{kg} / \mathrm{mm}^{2}\right) & \\
\tau_{\mathrm{a}}= & 0,5 \times 4,8 \mathrm{~kg} / \mathrm{mm}^{2} \\
= & 2,4 \mathrm{~kg} / \mathrm{mm}^{2}
\end{aligned}
$$

\section{c. Number of Nut Thread}

$$
\mathrm{Z} \geqq \frac{W}{\pi d_{2} H_{1} q_{a}}
$$

(Sularso's book p. 297 no.7.5)

where: $\mathrm{H}_{1}=$ height of hook $(\mathrm{mm})$

$$
\begin{aligned}
& \mathrm{Z}=\frac{1,82 \mathrm{~kg}}{3,14 \times 10,863 \mathrm{~mm} \times 0,947 m m \times 3 \mathrm{~kg} / \mathrm{mm} 2} \\
& =2
\end{aligned}
$$

\section{d. Height of Nut}

$\mathbf{H}=\mathbf{z} \cdot \mathbf{p}(\mathbf{m m})$

where: $\mathrm{H}=$ height of nut $(\mathrm{mm})$

standard: $\mathrm{H}=(0,8$ s.d 1,0$)$. $\mathrm{d}(\mathrm{mm})$

$\mathrm{p}=$ distance divider $(\mathrm{mm})$

(look at the table 7.1. a \& 7.1. b \& table

7.2 Sularso's book p 289 s.d 291)

$\mathbf{H}=2 \times 2,5 \mathrm{~mm}$

$=5 \mathrm{~mm}$

\section{e. Thread Root Shear Stress on Bolt}

$$
\tau_{b}=\frac{w}{\pi \cdot d_{1} \cdot k \cdot p \cdot z} \quad\left(\mathrm{~kg} / \mathrm{mm}^{2}\right)
$$

(Sularso's book p 297 no 7.8 )

where: $\tau_{b}=$ screw root shear stress on the bolt $\left(\mathrm{kg} / \mathrm{mm}^{2}\right)$

$$
\begin{gathered}
\mathrm{k} \cdot \mathrm{p}=\text { claw thick outer threaded } \\
\mathrm{k}=0,84
\end{gathered}
$$

$\tau_{\mathrm{b}}=\frac{1.82 \mathrm{~kg}}{3,14 \times 10,106 \mathrm{~mm} \times 0,84 \times 1,75 \mathrm{~mm} \mathrm{x} 2}$

$\tau_{\mathrm{b}}=2 \mathrm{~kg} / \mathrm{mm}^{2}$

\section{f. Thread Root Shear Stress in Nuts}

$$
\tau_{\mathrm{n}}=\frac{\mathrm{w}}{\pi . \text { D.j.p.z }} \quad\left(\mathrm{kg} / \mathrm{mm}^{2}\right)
$$

(Sularso's book p 297 no. 7.9)

where: $\quad \tau_{\mathrm{n}}=$ screw root shear stress on the nut $\left(\mathrm{kg} / \mathrm{mm}^{2}\right)$

$$
\mathrm{j}=0,75 \text { (metrical thread) }
$$

Sularso's book p. 297

$$
\begin{aligned}
\tau_{\mathrm{n}} & =\frac{1,82 \mathrm{~kg}}{3,14 \times 12 \mathrm{~mm} \times 0,75 \times 1,75 \mathrm{~mm} \times 2} \\
\tau_{\mathrm{n}} & =2 \mathrm{~kg} / \mathrm{mm}^{2}
\end{aligned}
$$

\section{Thread (Vise)}

Thread diameter $12 \mathrm{~mm}$ (steel S45C) coarse metric thread on the table

$\mathrm{d}_{1}=10,106 \mathrm{~mm}, \mathrm{~d}_{2}=10,863 \mathrm{~mm}$,

$\mathrm{D}=12 \mathrm{~mm}, \mathrm{p}=1,75 \mathrm{~mm}, \mathrm{H}_{1}=0,947$

$\mathrm{mm}$.

\section{a. Thread Tensile Stress}

$$
\sigma t=\frac{w}{A}=\frac{w}{\frac{\pi}{4} \cdot d 1^{2}}\left(\mathrm{~kg} / \mathrm{mm}^{2}\right)
$$

(Sularso's book p 296 no 7.1 )

where: $\sigma_{\mathrm{t}}=$ tensile stress $\left(\mathrm{kg} / \mathrm{mm}^{2}\right)$.

$$
\mathrm{W}=\text { axial tensile load on the }
$$
bolt $(\mathrm{kg})$.

$$
\begin{gathered}
\sigma t=\frac{W}{\frac{\mathbf{W}}{4} \cdot \mathbf{d 1 ^ { 2 }}} \\
=\frac{\mathbf{0 , 4} \mathbf{k g}}{\frac{3,14}{4} \cdot \mathbf{1 0}, \mathbf{1 0 6} \mathbf{~ m m}^{2}} \\
=0,005 \mathrm{~kg} / \mathrm{mm}^{2}
\end{gathered}
$$

\section{b. Shear Stress Permission}

$$
\tau_{\mathrm{a}}=\left(\begin{array}{llll}
0,5 \mathrm{~s} . \mathrm{d} & 0,75) & \sigma_{\mathrm{a}} & \left(\mathrm{kg} / \mathrm{mm}^{2}\right)
\end{array}\right.
$$

(Sularso's book p 297)

where: $\tau_{\mathrm{a}}=$ shear stress $\left(\mathrm{kg} / \mathrm{mm}^{2}\right)$

$$
\begin{aligned}
& \tau_{\mathrm{a}}=0,5 \times 4,8 \mathrm{~kg} / \mathrm{mm}^{2} \\
& =2,4 \mathrm{~kg} / \mathrm{mm}^{2}
\end{aligned}
$$




\section{c. Number of Nut Threads}

$$
\mathrm{Z} \geqq \frac{W}{\pi d_{2} H_{1} q_{a}}
$$

(Sularso's book p 297 no.7.5 )

where : $\mathrm{H}_{1}=$ height of hook $(\mathrm{mm})$

$$
\begin{aligned}
\mathbf{Z} & =\frac{0,4 \mathrm{~kg}}{3,14 \times 10,863 \mathrm{~mm} \times 0,947 \mathrm{~mm} \times 3 \mathrm{~kg} / \mathrm{mm} 2} \\
& =0,004
\end{aligned}
$$

\section{d. Height of Nut}

$$
\mathbf{H}=\mathbf{z} \cdot \mathbf{p}(\mathbf{m m})
$$

where: $\mathrm{H}=$ height of nut (mm)

standadr: $H=(0,8$ s.d 1,0$)$. $d(\mathrm{~mm})$

$\mathrm{p}=$ distance divider $(\mathrm{mm})$

(look at the table of 7.1. a \& 7.1. b \& table

7.2 Sularso's book p 289 s.d 291)

$$
\begin{aligned}
\mathbf{H} & =2 \times 2,5 \mathrm{~mm} \\
& =0,007 \mathrm{~mm}
\end{aligned}
$$

\section{e. Thread Root Shear Stress on Bolt}

$$
\tau_{b}=\frac{w}{\pi \cdot d_{1} \cdot k \cdot p \cdot z} \quad\left(\mathrm{~kg} / \mathrm{mm}^{2}\right)
$$

(Sularso's book p 297 no 7.8 )

where: $\quad \tau_{b}=$ thread root shear stress on bolt $\left(\mathrm{kg} / \mathrm{mm}^{2}\right)$

$\mathrm{k} . \mathrm{p}=$ claw thick outer threaded

$$
\mathrm{k}=0,84
$$

$$
\begin{aligned}
\tau_{b} & =\frac{0,4 \mathrm{~kg}}{3,14 \times 10,106 \mathrm{~mm} \times 0,84 \times 1,75 \mathrm{~mm} \times 2} \\
\tau_{\mathbf{b}} & =2 \mathrm{~kg} / \mathrm{mm}^{2}
\end{aligned}
$$

\section{f. Thread Root Shear Stress in Nuts}

$$
\tau_{\mathrm{n}}=\frac{\mathrm{w}}{\pi . \text {.j.j.p.z }} \quad\left(\mathrm{kg} / \mathrm{mm}^{2}\right)
$$

(Sularso's book p 297 no. 7.9)

where: $\quad \tau_{n}=$ screw root shear stress on the nut $\left(\mathrm{kg} / \mathrm{mm}^{2}\right)$

$\mathrm{j}=0.75$ (metrical thread) Sularso's book p 297

$$
\begin{aligned}
\tau_{\mathrm{n}} & =\frac{0,4 \mathrm{~kg}}{3,14 \times 12 \mathrm{~mm} \times 0,75 \times 1,75 \mathrm{~mm} \times 2} \\
\tau_{\mathrm{n}} & =2 \mathrm{~kg} / \mathrm{mm}^{2}
\end{aligned}
$$

\section{CONCLUSION}

Based on the results of research and discussion, it can be concluded that:

1. Jig \& Fixture is a tool that functions to help job seekers are faster, easier, and have good accuracy.

2. The design of this Jig \& Fixture can increase engineering, productivity, and ensure the quality of product results, as well as provide efficiency and lower costs on the manufacture of machinery.

\section{REFERENCES}

Harsono Wiryosumarto, 2004, Teknologi Pengelasan logam, PT Pradya Paramita, Jakarta.

Hoffman, Edward G., 1996, Jig and Fixture Design, Delmar Publishers.

Sularso, Kiyokatsu Suga, Dasar Perencanaan dan Pemilihan Elemen Mesin PT Pradya Paramita, Jakarta, 1991.

Aa Santosa. Jurnal Perancangan Jig dan Fixture Sistem Pneumatik untuk proses pemasangan Bearing dan Absorber padaVelg Rear Wheel, 2017.

Muh Alfatih Hendrawan, Pramuko Ilmu Purbopotro. Jurnal Rancang Bangun Jig Drilling Sebagai Solusi Pembuatan Lubang Chassis Minitruk yang di produksi, 2016

Ft.unsada.ac.id/wpcontent/uploads/2008/04/ba b9-pp2.pdf 